\title{
Diffusion and Relaxation Dynamics in Cluster Crystals
}

\author{
Angel J. Moreno ${ }^{1,2}$ and Christos N. Likos ${ }^{1}$ \\ ${ }^{1}$ Institut für Theoretische Physik II: Weiche Materie, Heinrich-Heine-Universität Düsseldorf, D-40225 Düsseldorf, Germany \\ ${ }^{2}$ Centro de Física de Materiales (CSIC-UPV/EHU), Apartado 1072, E-20080 San Sebastián, Spain
}

(Received 5 June 2007; published 7 September 2007)

\begin{abstract}
For a large class of fluids exhibiting ultrasoft bounded pair potentials, particles form crystals consisting of clusters located in the lattice sites, with a density-independent lattice constant. Here we present an investigation on the dynamic features of a representative example of this class. It is found that particles can diffuse between lattice sites, maintaining the lattice structure, through an activated hopping mechanism. This feature yields finite values for the diffusivity and full relaxation of density correlation functions. Simulations suggest the existence of a localization transition which is avoided by hopping and a dynamic decoupling between self- and collective correlations.
\end{abstract}

PACS numbers: 61.20.Ja, 64.70.Pf, 82.70.-y

The investigation of large-scale structural and dynamic properties of complex fluids can be facilitated by coarsegraining intramolecular fast degrees of freedom. By following this procedure, each macromolecule is represented as a single particle interacting with any other through an effective ultrasoft pair potential [1], which for isotropic interactions just depends on the distance between the centers of mass. The effective potential is bounded if centers of mass can coincide without violating excluded volume conditions. Some examples are polymer chains [2], dendrimers [3], or microgels [4].

Generalized exponential models (GEM), $v(r)=$ $\epsilon \exp \left[-(r / \sigma)^{m}\right]$, constitute a class of such effective bounded interactions. The cases $m \leq 2$ and $m>2$ belong, respectively, to the so-called $Q^{+}$and $Q^{ \pm}$classes for which the Fourier transform, $\tilde{v}(q)$, of $v(r)$ is, respectively, positive or oscillating around zero. According to a general criterion [5], systems belonging to the $Q^{+}$class display reentrant crystallization in the density-temperature plane $[6,7]$ whereas potentials of the $Q^{ \pm}$class rather display a monotonic freezing line beyond which the system forms cluster crystals [5,8-10]. These are novel forms for the self-organization of (soft) matter [11] because they feature a lattice constant that is density independent and the cluster population is proportional to the density $[5,9,10]$. Recent simulations of amphiphilic dendrimers show that the latter indeed interact via a $Q^{ \pm}$potential [12]. Very similar phenomena appear for the case of two-dimensional, hard disksquare shoulder potentials [13].

Previous theoretical and computational investigations of the GEM model for $m>2$ have focused on structural and thermodynamic properties $[9,10]$. A detailed study of the dynamics of these crystals is still missing and their particular form of self-organization suggests that there must exist strong differences from that of atomic solids with single site occupancy. The purpose of this Letter is to investigate the novel features arising in the dynamics of such cluster crystals in general. We find that particles diffuse between lattice sites through an activated hopping mechanism, without breaking the lattice structure, resulting in finite values for the diffusivity and full relaxation of self-correlation functions. We establish the existence of a localization transition which is avoided by hopping and is distinct for self- and density-density correlations. Therefore, an unusual decoupling between self- and collective dynamics is observed, the latter exhibiting a lower transition temperature than the former.

Particles in the simulated system interact through a GEM potential with $m=8$, which is cut off at a distance $r_{c}=1.5 \sigma$. The particle mass $m_{p}$, energy scale $\epsilon$, and particle diameter $\sigma$ are set to one. In the following, energy, temperature, density, time, distance, and wave vector will be given, respectively, in units of $\epsilon, \epsilon / k_{B}, \sigma^{-3}$, $\sigma\left(m_{p} / \epsilon\right)^{1 / 2}, \sigma$, and $\sigma^{-1}$. We investigate dynamic features of the cluster crystals, in a wide range of temperature $T$, deep inside the region of stability of the fcc phase $[9,10]$. The investigated densities are $\rho=N / L^{3}=2.0,3.0,4.0$, and 7.0. The values of the cell size, $L$, used for periodic boundary conditions are integer multiples of the $\rho$-independent lattice constant $a$. Following $[9,10]$, for a fcc lattice $a=2 \pi \sqrt{3} / q_{*}$, with $q_{*}$ the wave vector for which $\tilde{v}(q)$ shows its first minimum. We obtain $a=$ 1.8571325 . The distance between nearest-neighbor lattice sites is $d_{\mathrm{nn}}=a / \sqrt{2}=1.313191$. Because of the large values of $\rho$, investigation of relaxation dynamics in the present system is computationally more demanding that for standard model systems with unbounded interactions as, e.g., Lennard Jones mixtures [14], which are typically studied at $\rho \sim 1.0$. Thus, Newtonian rather than Brownian dynamics simulations have been carried out, as the latter would yield a much slower relaxation [15].

Figure 1 shows the $T$ dependence of the mean squared displacement for densities $\rho=2.0$ and $\rho=7.0$. The initial ballistic regime corresponds to oscillations around lattice sites whose period scales, according to [10], as $\rho^{-1 / 2}$. Rescaling time as $\rho^{1 / 2} t$ causes a collapse of the curves at short times and confirms the above result that was 


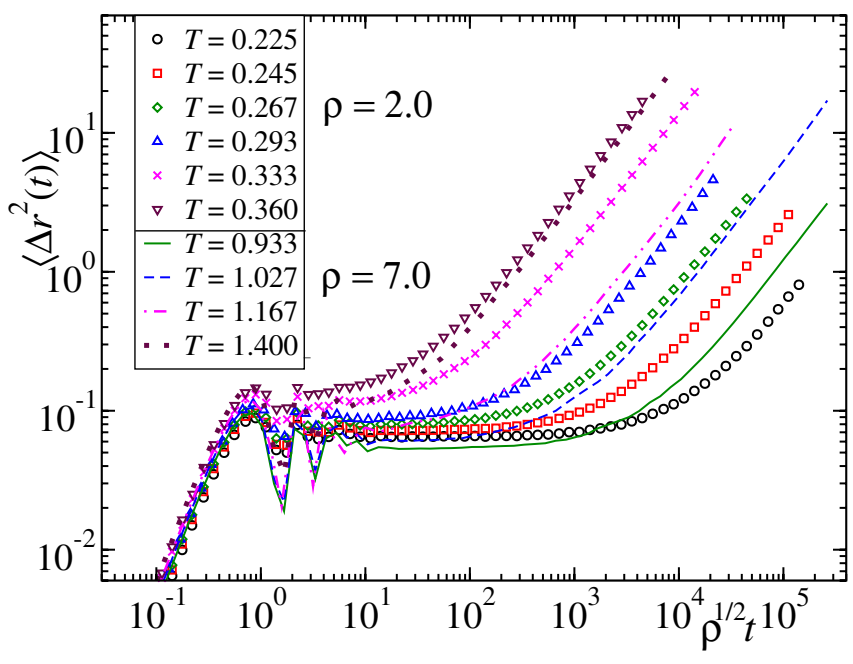

FIG. 1 (color online). Temperature dependence of the mean squared displacement for density $\rho=2.0$ (symbols) and 7.0 (lines).

derived on the basis of statics [10]. Short-time motion is dictated, thus, by $\rho$ alone whereas long-time diffusion is dictated by $\rho / T$, as we will shortly demonstrate. After the ballistic regime, a plateau arises characterizing the temporary trapping of the particles within the clusters. At long times, the particles reach the diffusive regime $\left(\left\langle\Delta r^{2}(t)\right\rangle \sim\right.$ $t)$ at all the investigated temperatures, reaching distances of at least one particle diameter from their initial position. The fcc structure remains stable in the whole time window of the simulation. Therefore, particles move between neighboring clusters, modifying thus the clusters' initial identity but leaving unaffected their average population $n_{c}$.

Figure 2 displays an illustrative example of the time evolution of the van Hove self-correlation function, $G_{s}(r, t)$. The discrete nature of the motion between clusters centered around distinct lattice sites is evidenced by the succession of peaks arising in $G_{s}(r, t)$. With increasing time, the height of the first peak progressively decreases and new peaks of increasing intensity located at larger distances arise. From integration of $G_{s}(r, t)$ for the longest represented $t$ it is found that more than $80 \%$ of the particles are located at distance $r>0.5 d_{\mathrm{nn}}$ from their initial position; i.e., they have moved to different lattice sites. Though a detailed characterization of jumps between lattice sites is beyond the scope of this Letter, it is worth commenting some interesting features of $G_{s}(r, t)$. Arrows in Fig. 2 indicate distances between $n$th nearest-neighbor lattice sites, $\sqrt{n} d_{\mathrm{nn}}$, where $n=1,2,3$, and 4 . The two first sharp maxima after the initial peak match such distances for $n=$ 1 and 3. The pronounced minimum at $r \cong 1.4 d_{\text {nn }}$ matches the case $n=2$. Given two consecutive jumps connecting three distinct lattice sites $1,2,3$ for which $d_{12}=d_{23}=$ $d_{\mathrm{nn}}$, it is easy to see that the four allowed angles between both jumps, $60^{\circ}, 90^{\circ}, 120^{\circ}$, and $180^{\circ}$ yield, respectively, $d_{13} / d_{\mathrm{nn}}=1, \sqrt{2}, \sqrt{3}$, and 2 . The presence of a marked

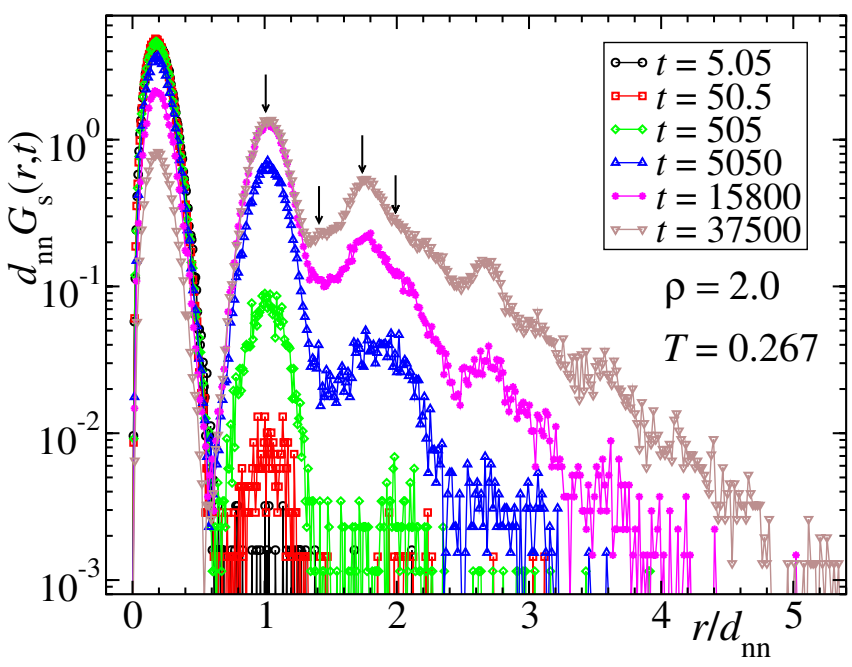

FIG. 2 (color online). The van Hove self-correlation function, $d_{\mathrm{nn}} G_{s}(r, t)$, computed at different times, vs distance $r / d_{\mathrm{nn}}$, where $d_{\mathrm{nn}}$ is the distance between nearest-neighbor lattice sites, at $\rho=$ 2.0 and $T=0.267$. Arrows correspond to distances between $n$ thneighbor lattice sites, for $n=1,2,3$, and 4 .

minimum for $r / d_{\mathrm{nn}}=\sqrt{2}$ and a sharp maximum for $r / d_{\mathrm{nn}}=\sqrt{3}$ suggests a preferential directionality for the motion between neighboring sites with low and high probability for angles of, respectively, $90^{\circ}$ and $120^{\circ}$ between consecutive jumps.

A map of the local potential energy values $U$, which would be experienced by a test particle in the system, shows minima on the lattice sites and maxima at the interstitials, confirming the one-dimensional result of Fragner [16]. The potential barriers between nearestneighbor minima are much lower than those between next-nearest ones, suggesting preferential hops to nearest neighbors, in agreement with the results for $G_{s}(r, t)$ in Fig. 2. The distribution of local $U$ values computed over the whole simulation box, $g_{t}(U)$, is shown in Fig. 3(a) and only on the lattice sites, $g_{l}(U)$, in Fig. 3(b). The distribution $g_{l}(U)$ is dominated by the contribution of the cluster centered around the considered site. Since the number of particles forming the cluster is polydisperse and discrete, $g_{l}(U)$ shows a peak structure. Consistently with the invariance of lattice constant and the relation $n_{c} \propto \rho$, approximate $\rho$-scaling behavior is observed for $g_{t}(U)$, of increasing quality at higher densities. The maximum at $U_{t} \cong 4 \rho$ corresponds to values at interstitials between nearest-neighbor sites. Though the most probable $U$ for $g_{l}(U)$ shows a weak $\rho$ dependence, we take a "common" value $U_{l} \cong 1.7 \rho$ as a rough estimate of the average local minimum at the lattice sites, obtaining the typical barrier separating nearest-neighbor lattice sites as $\Delta U \equiv$ $U_{t}-U_{l} \cong 2.3 \rho$.

Figure 4 displays results for the diffusivity $D$, obtained as the long-time limit of $\left\langle\Delta r^{2}(t)\right\rangle / 6 t$. The $\rho$ and $T$ dependence of $D$ can be understood as follows. Each particle 

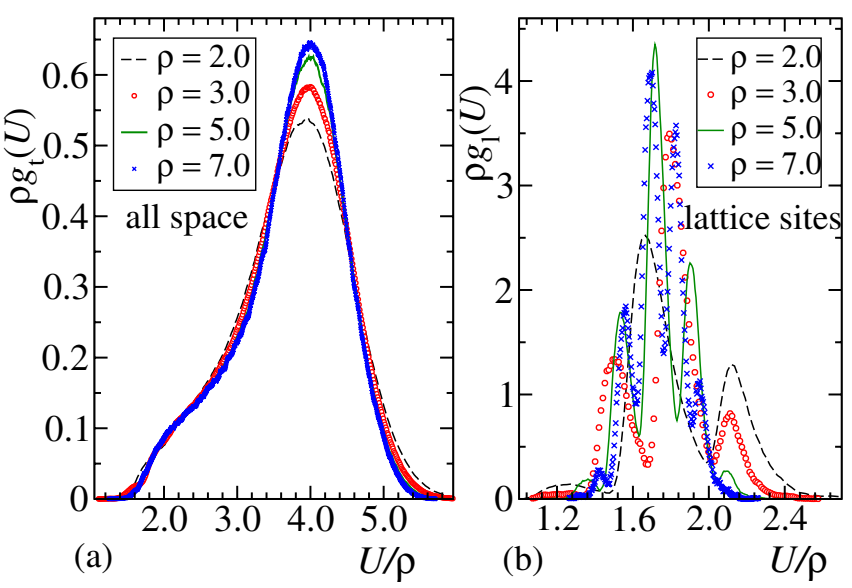

FIG. 3 (color online). The distribution of local potential energy, $\rho g(U)$, vs rescaled energy, $U / \rho$, at different densities. (a) Results considering all the volume in the simulation box; (b) results by computing $U$ only at lattice sites.

located on any given lattice site experiences a harmonic potential with a barrier height $\Delta U$ to the neighboring site, as mentioned above. Because of the contact with the heat bath, the energy $E$ of a particle follows the distribution $p(E)=e^{-\beta E}\left(\beta^{3} E^{2}\right) / 2$ for $E>0$ and vanishing for $E<0$, with $\beta=\left(k_{B} T\right)^{-1}$. The probability $P_{>}(\Delta U)$ to have $E>$ $\Delta U$ can be calculated as $P_{>}(z)=\int_{z}^{\infty} p(E) d E=$ $e^{-\beta z}\left[(\beta z)^{2} / 2+\beta z+1\right]$. Once $E>\Delta U$, the particle hops to the neighboring site. We can now translate $P_{>}(\Delta U)$ into a "waiting time" that particles typically spend on a given lattice site before hopping to the next one. This time scales as $\sim 1 / P_{>}(\Delta U)$. If we observe any given particle for a number of time steps $N_{s} \gg 1$, there will only be a fraction $N_{\text {hop }} \sim P_{>}(\Delta U) N_{s}$ of them for which the particle will hop, its motion being a random

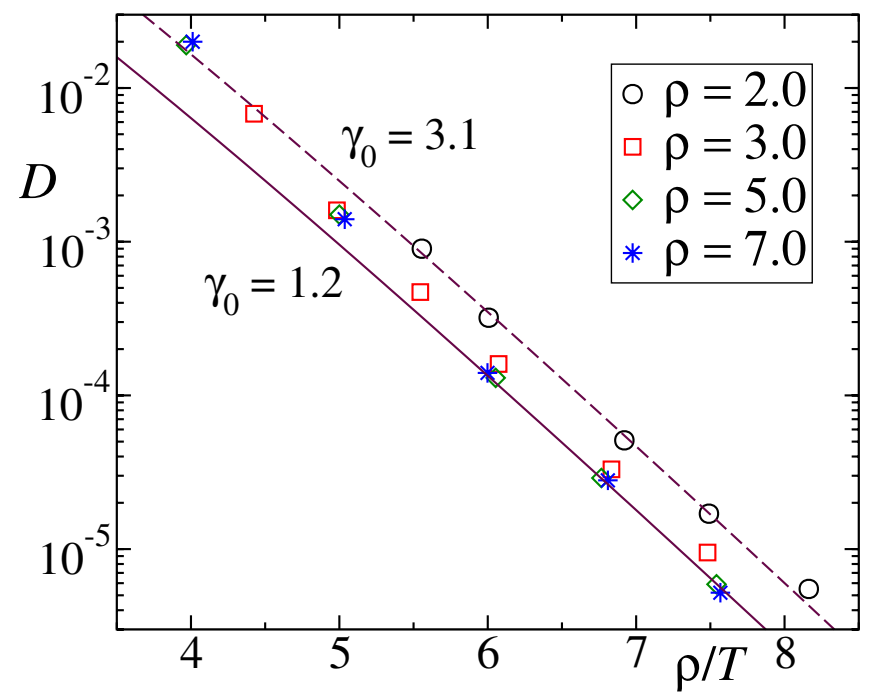

FIG. 4 (color online). Temperature dependence of the diffusivity for different densities. Lines are fits to Eq. (1); the values of the fit parameter $\gamma_{0}$ are indicated on the plot. walk of step size $\ell \sim \sigma$. Hence, measured in the natural units of the problem, $\left\langle\Delta r^{2}(t)\right\rangle$ will scale as $P_{>}(\Delta U) t$. By using $\Delta U \cong 2.3 \rho$ (see above) we obtain

$$
D=\gamma_{0}\left[(2.3 \rho / T)^{2} / 2+2.3 \rho / T+1\right] e^{-2.3 \rho / T},
$$

with some numerical coefficient $\gamma_{0}$ of order unity. Hence, theory predicts that $D$ depends solely on the ratio $\rho / T$. The approximations involved in deriving Eq. (1) become more accurate as $\rho$ grows, for which case the polydispersity in the cluster population is reduced and all sites can be treated as identical harmonic wells. Results in Fig. 4 fully corroborate this treatment. The diffusivity can be very well described by Eq. (1) and indeed for sufficiently high $\rho$ all data points collapse on a single curve, where $D$ is expressed as a function of the ratio $\rho / T$. This is a dynamical generalization of the scaling properties previously found for statics [10].

Finally, we discuss self- and density-density correlators of wave vector $q$, respectively, defined as $F_{s}(q, t)=$ $N^{-1}\left\langle\sum_{j} \exp \left\{i \mathbf{q} \cdot\left[\mathbf{r}_{j}(t)-\mathbf{r}_{j}(0)\right]\right\}\right\rangle, \quad$ and $\quad F(q, t)=$ $\left\langle\rho_{q}(t) \rho_{q}^{*}(0)\right\rangle /\left\langle\rho_{q}(0) \rho_{q}^{*}(0)\right\rangle, \quad$ with $\quad \rho_{q}(t)=\sum_{j} \exp [i \mathbf{q} \cdot$ $\mathbf{r}_{j}(t)$ ]. Figure 5 shows, for $\rho=2.0$ and for a fixed wave vector $q=4.0$ not probing the lattice structure, the $T$ dependence of $F_{s}(q, t)$ and $F(q, t)$. After the first microscopic decay, both correlators exhibit a plateau, whose duration increases with decreasing $T$. As usually found in systems displaying slow relaxation, the presence of this plateau indicates a temporary freezing of such correlations. Strong density oscillations related with intracluster motion are observed at the crossover between the microscopic and plateau regimes [17]. At long times self-correlations relax and decay to zero. This is also true for density-density correlations if $q$ does not match any wave vector probing the lattice structure. On the contrary, and consistently with the observed stability of the fcc lattice, density-density correlations for $q$ belonging to the reciprocal lattice are permanently frozen, and there is no signature of a final decay of the plateau, which remains flat (not shown) up to the limit of the simulation window.

An ideal (i.e., without intervening hopping events) localization transition to a nonergodic ("glassy") phase can be defined by a jump from zero to a finite value (nonergodicity parameter) of the long-time limit of dynamic correlators [18]. Hence, at the transition point the nonergodicity parameter is equal to the plateau height. This quantity progressively increases with decreasing $T$ in the nonergodic phase. The presence of hopping events can, in principle, restore ergodicity at temperatures below the ideal transition, leading to final relaxation of correlators [18]. Still, the existence of an avoided ideal transition can still be identified by the increase of the plateau height. Now we investigate features of such a transition in the present system. As shown in Fig. 5, $F_{s}(q, t)$ and $F(q, t)$ exhibit a rather different behavior at the intermediate plateau regime. The plateau height, $f_{q}^{s}$, for $F_{s}(q, t)$ increases with 

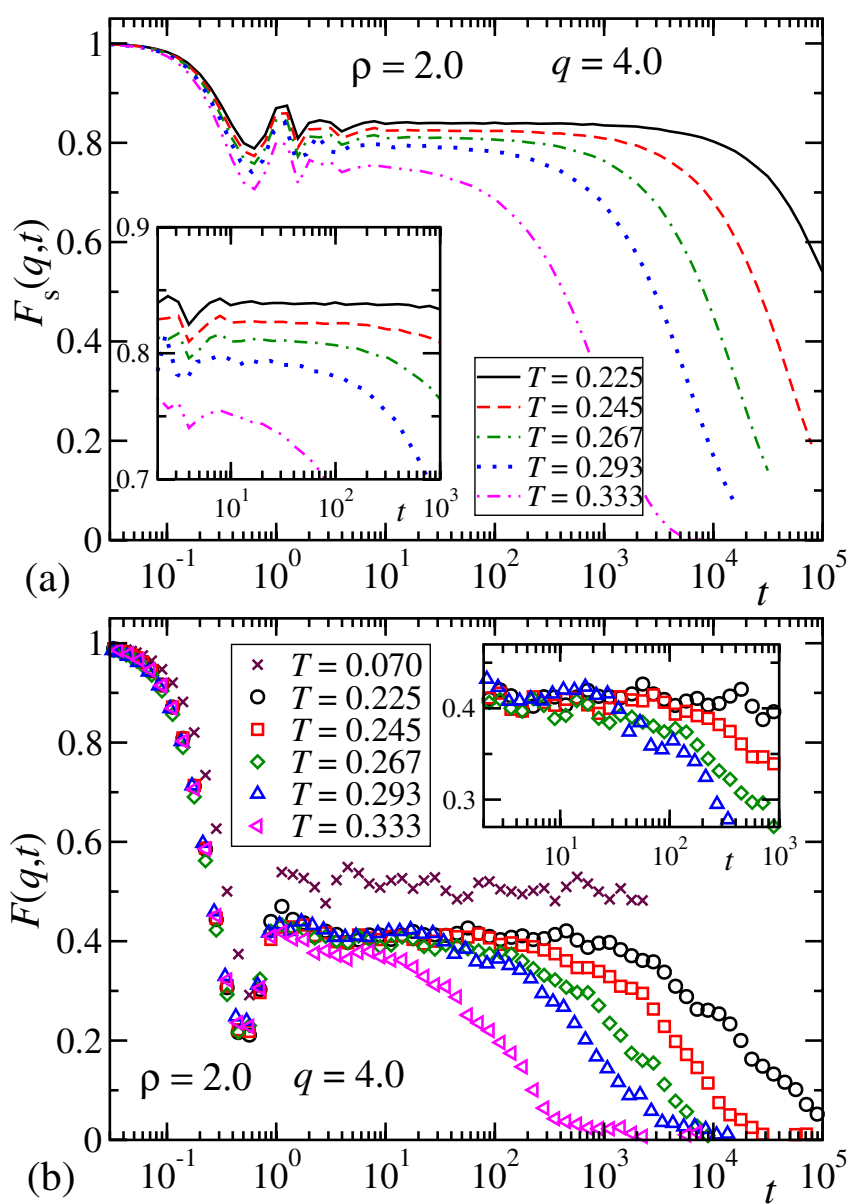

FIG. 5 (color online). Temperature dependence of $F_{s}(q, t)$ (a) and $F(q, t)(\mathrm{b})$, for $\rho=2.0$ and $q=4.0$. The insets show a magnification of the plateau regime.

decreasing $T$ [see inset in Fig. 5(a)], resulting in a broader width of $f_{q}^{s}$ that indicates a decrease of the localization length [18]. This feature is observed for all wave vectors and suggests the existence of a localization transition for self-motions. However, in contrast to the standard behavior in crystal states, and similarly to relaxation in supercooled fluids, hopping events move the particles beyond the localization length and ergodicity for self-motions is restored, yielding final relaxation of $F_{s}(q, t)$.

The inset in Fig. 5(b) shows that, in the same $T$ range of the latter data, the plateau height, $f_{q}$, for $F(q, t)$ stays constant ( $T$ independent), contrary to the case of $f_{q}^{s}$. The values of $f_{q}$ are notably smaller than those of $f_{q}^{s}$, indicating a weaker localization for collective motions. This behavior is indeed observed for all $q$ values, except for those probing the lattice structure, for which a progressive increase of the plateau is observed with decreasing $T$ (not shown). An increase of $f_{q}$ for $q$ not probing the lattice structure is only observed at much lower $T$ [see data for $T=0.070$ in Fig. 5(b)]. Though for these low- $T$ values the system cannot be equilibrated and aging effects are observed at long times (not shown), such effects do not affect the behavior of $F(q, t)$ at intermediate times, where the plateau regime arises. Results in Fig. 5(b) show that the localization transition occurs at lower $T$ for out-of-lattice collective correlations than for self-correlations; i.e., there is a dynamic decoupling between self- and collective relaxation. These results resemble dynamic features of plastic crystals, where molecules are constrained to vibrate around lattice positions, but can perform full rotations leading to relaxation of out-of-lattice collective correlations (in the present case cluster deformation is an additional mechanism). However, contrary to the case of plastic crystals, activated hopping restores ergodicity of translational motions and leads to finite diffusivity and full decay of selfcorrelators.

In summary, we have investigated slow dynamics in the cluster crystal phase of a representative model of macromolecules in solution interacting through effective ultrasoft bounded potentials. The obtained major features are a full change of the initial identity of the clusters through particle hopping between lattice sites and dynamic decoupling between self- and collective out-of-lattice correlations.

We thank B. M. Mladek, P. Charbonneau, H. Fragner, and D. Frenkel for useful discussions. Financial support from SoftComp No. NMP3-CT-2004-502235 and DIPCSpain is acknowledged.

[1] C. N. Likos, Phys. Rep. 348, 267 (2001).

[2] A. A. Louis et al., Phys. Rev. Lett. 85, 2522 (2000).

[3] I. O. Götze et al., J. Chem. Phys. 120, 7761 (2004).

[4] A. R. Denton, Phys. Rev. E 67, 011804 (2003); 68, 049904(E) (2003).

[5] C. N. Likos et al., Phys. Rev. E 63, 031206 (2001).

[6] A. Lang et al., J. Phys. Condens. Matter 12, 5087 (2000); S. Prestipino et al., J. Chem. Phys. 123, 144110 (2005).

[7] D. Gottwald et al., Phys. Rev. Lett. 92, 068301 (2004).

[8] C. N. Likos et al., Phys. Rev. E 58, 3135 (1998).

[9] B. M. Mladek et al., Phys. Rev. Lett. 96, 045701 (2006); 97, 019901(E) (2006).

[10] C. N. Likos et al., J. Chem. Phys. 126, 224502 (2007).

[11] D. Frenkel, Nature (London) 440, 4 (2006).

[12] B. M. Mladek, G. Kahl, and C.N. Likos, cond-mat/ 0708.4380 .

[13] M. A. Glaser et al., Europhys. Lett. 78, 46004 (2007).

[14] T. Gleim et al., Phys. Rev. Lett. 81, 4404 (1998).

[15] Though Brownian dynamics provide a more realistic picture at short times, Newtonian and Brownian dynamics are equivalent within a time shift at long-time scales probing slow relaxation [14].

[16] H. Fragner, Phys. Rev. E 75, 061402 (2007).

[17] In principle, the inclusion of solvent effects through Brownian dynamics would damp these oscillations.

[18] W. Götze and L. Sjögren, Rep. Prog. Phys. 55, 241 (1992). 